\title{
Erratum to: Design of Mn porphyrins for treating oxidative stress injuries and their redox-based regulation of cellular transcriptional activities
}

Ines Batinic-Haberle • Ivan Spasojevic $\cdot$ Hubert M. Tse • Artak Tovmasyan - Zrinka Rajic - Daret K. St. Clair • Zeljko Vujaskovic · Mark W. Dewhirst · Jon D. Piganelli

Published online: 24 December 2010

(C) Springer-Verlag 2010

\section{Erratum to: Amino Acids}

DOI 10.1007/s00726-010-0603-6

In the original version of Fig. 4, the two bars representing SOD deficient and SOD proficient in "Aerobic Growth of E. coli" were misplaced. The corrected figure is produced in the following page. In Fig. 4 legend, "cytosolic $\mathrm{Cu}, \mathrm{ZnSOD}$ " is corrected to "cytosolic SODs".

The online version of the original article can be found under doi:10.1007/s00726-010-0603-6.

I. Batinic-Haberle $(\bowtie) \cdot$ A. Tovmasyan · Z. Rajic .

Z. Vujaskovic · M. W. Dewhirst

Department of Radiation Oncology, Duke University Medical

Center, 281b/285 MSRB I, Durham, NC 27710, USA

e-mail: ibatinic@duke.edu

I. Spasojevic

Department of Medicine, Duke University Medical Center,

Durham, NC 27710, USA

H. M. Tse

Department of Microbiology, Comprehensive Diabetes Center Birmingham, University of Alabama at Birmingham,

Birmingham, AL 35294, USA

D. K. St. Clair

Graduate Center for Toxicology, University of Kentucky,

Lexington, KY 40536, USA

J. D. Piganelli

Children's Hospital, Rangos Research Center,

University of Pittsburgh, Pittsburgh, PA 15213, USA 


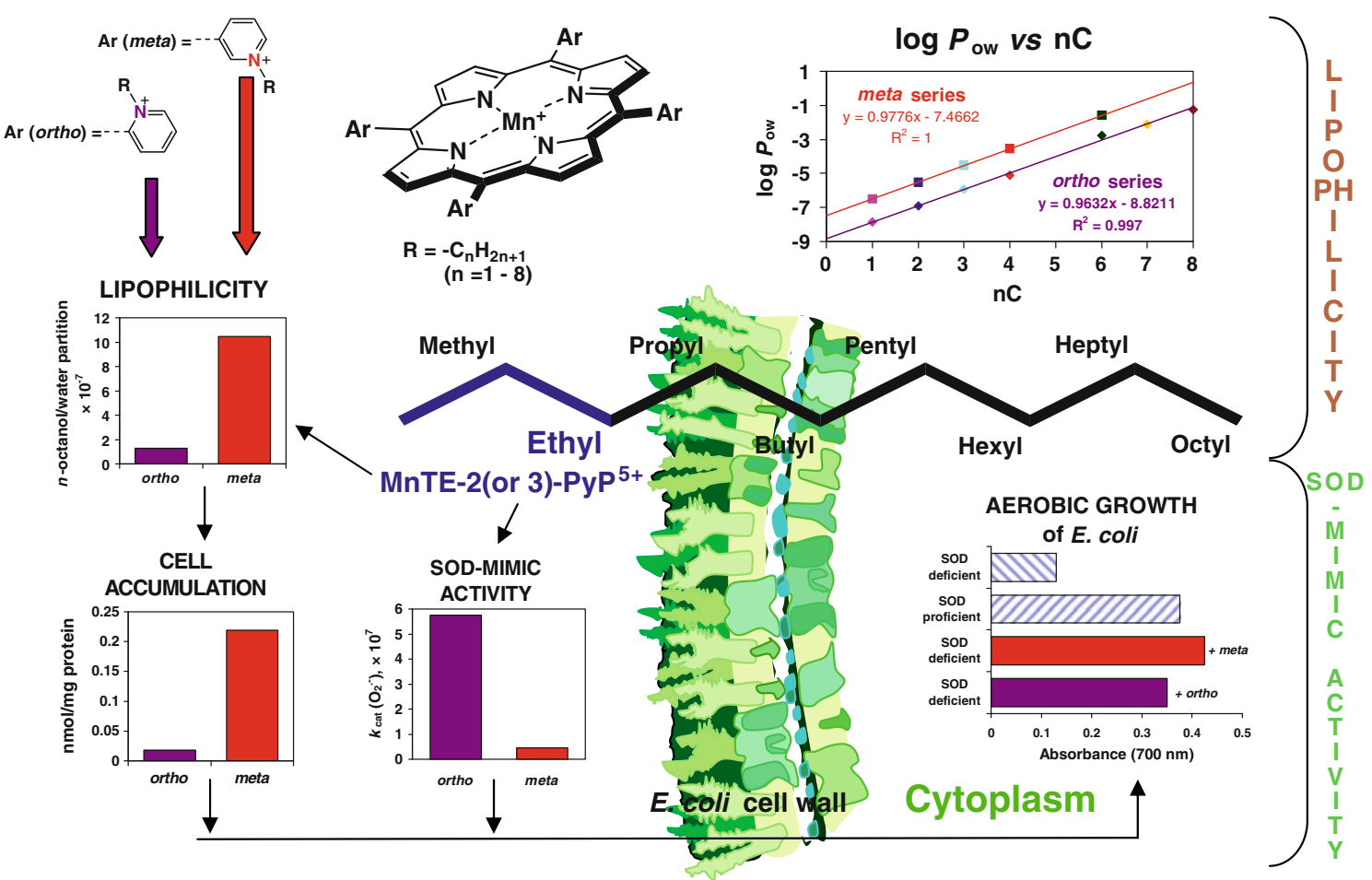

Fig. 4 Lipophilicity of MnPs increases tenfold by either (1) lengthening alkyl chains by each additional carbon atom, or (2) shifting alkyl groups from ortho (2) to meta (3) positions. The tenfold increased lipophilicity of meta ethyl analog, MnTE-3-PyP $\mathrm{P}^{5+}$, resulted in its tenfold higher accumulation in the cytosol of E. coli as compared to ortho isomer, MnTE-2-PyP ${ }^{5+}$. Such enhanced accumulation compensated for a tenfold lower ability of MnTE-3-PyP ${ }^{5+}$ to dismute $\mathrm{O}_{2}^{--}$. In turn both isomers were equally able to substitute for the lack of cytosolic SODs when E. coli grew in aerobic medium (Kos et al. 2009a, b) 